\title{
Association between the first two immunoglobulin-like domains of the neural cell adhesion molecule N-CAM
}

\author{
Annette R. Atkins ${ }^{\mathrm{a}, 1}$, Michael J. Osborne ${ }^{\mathrm{a}, 1}$, Hilal A. Lashuel ${ }^{\mathrm{b}}$, Gerald M. Edelman ${ }^{\mathrm{c}, \mathrm{d}}$, \\ Peter E. Wright ${ }^{\mathrm{a}, \mathrm{d}}$, Bruce A. Cunningham ${ }^{\mathrm{c}}$, H. Jane Dyson ${ }^{\mathrm{a}, *}$ \\ a Department of Molecular Biology, The Scripps Research Institute, 10596 North Torrey Pines Rd, La Jolla, CA 92037, USA \\ ${ }^{\mathrm{b}}$ Department of Chemistry, The Scripps Research Institute, 10596 North Torrey Pines Rd, La Jolla, CA 92037, USA \\ ${ }^{\mathrm{c}}$ Department of Neurobiology, The Scripps Research Institute, 10596 North Torrey Pines Rd, La Jolla, CA 92037, USA \\ ${ }^{\mathrm{d}}$ Skaggs Institute for Chemical Biology, The Scripps Research Institute, 10596 North Torrey Pines Rd, La Jolla, CA 92037, USA
}

Received 2 March 1999; received in revised form 8 April 1999

\begin{abstract}
The extracellular domain of N-CAM contains five immunoglobulin-like (Ig) and two fibronectin type III-like domains and facilitates cell-cell binding through multiple, weak interdomain interactions. NMR spectroscopy indicated that the two N-terminal Ig-like domains from chicken N-CAM (Ig I and Ig II) interact with millimolar affinity. Physico-chemical studies show that this interaction is significantly amplified when the domains are covalently linked, consistent with an antiparallel domain arrangement. The binding of the two individual domains and the dimerization of the concatenated protein were essentially independent of salt, up to a concentration of $200 \mathrm{mM}$. The residues in Ig $I$ involved in the interaction map to the BED strands of the $\beta$ sandwich, and delineate a largely hydrophobic patch.
\end{abstract}

(C) 1999 Federation of European Biochemical Societies.

Key words: N-CAM; Homophilic binding; Ig fold; Nuclear magnetic resonance

\section{Introduction}

The neural cell adhesion molecule, N-CAM, has been shown to be capable of both homophilic and heterophilic binding, and has been implicated in a variety of physiological processes including neurite fasciculation, the formation of neuromuscular junctions, and neuron-glia interactions. The extracellular region of N-CAM is composed of five immunoglobulin-like (Ig-like) domains and two regions with homology to the fibronectin type III (FnIII) repeats. This modular structure is characteristic of $\mathrm{Ca}^{2+}$ independent cell adhesion molecules, where varying numbers of Ig-like and FnIII-like repeats are combined [1].

Early studies using monoclonal antibodies and N-CAM fragments indicated that homophilic binding involved Ig IIV [2-4] and particularly Ig I-II and Ig III [2]; the fifth domain, which contains polysialic acid, could influence binding but did not appear essential [3]. Others found that all five Ig domains were required for N-CAM mediated adhesion [5].

\footnotetext{
*Corresponding author. Fax: (1) (619) 7849822.

E-mail: dyson@scripps.edu

1 These authors contributed equally to this work.
}

Abbreviations: CD, circular dichroism; HSQC, heteronuclear single quantum coherence; N-CAM, neural cell adhesion molecule; FnIII, fibronectin type III repeats; Ig I-V, immunoglobulin-like domains I$\mathrm{V}$ respectively; $s$, sedimentation coefficient; S, Svedberg unit of $10^{-13}$ $\mathrm{s} ; D$, diffusion coefficient; F, Ficks diffusion unit of $10^{-7} \mathrm{~cm}^{2} \mathrm{~s}^{-1}$
Further work using soluble, recombinant Ig-like domains attached to beads suggested a model of N-CAM:N-CAM binding that involved an antiparallel interaction of the five $\mathrm{Ig}$ domains [6]. In contrast, binding between Ig I and Ig II was demonstrated using surface plasmon resonance, and specific interactions between other Ig domains were not found [7].

Stable cell adhesion results from the interactions of large numbers of adhesion molecules concentrated at the cell-cell or cell-matrix interface. The interactions of individual cell adhesion molecules are weak, which has generally precluded the direct study of the bound adhesion complex. However, low affinity interactions are amenable to NMR studies, where the combination of millimolar protein concentrations, a time averaged analysis and the unique sensitivity and site specificity of the NMR chemical shift facilitates observation of specific weak interactions. Perturbations in chemical shifts have been used to study the micromolar interaction of soluble Ig-like domains from CD2 and CD48, where chemical shift changes and differential line broadening were used to map the binding site [24]. Similarly, the millimolar binding of a small peptide from CD4 to the Nef protein was mapped onto the Nef structure on the basis of peptide-induced chemical shift changes [25].

Here we present evidence derived from NMR studies that the first two N-terminal Ig-like domains of N-CAM associate with millimolar affinity and map the interaction to a largely hydrophobic surface on the N-terminal Ig-like domain, Ig I. Analytical ultracentrifugation studies demonstrated that the observed interaction between Ig I and Ig II is significantly amplified when the two domains are covalently linked in a contiguous fashion, as they are in the native N-CAM protein.

\section{Materials and methods}

\subsection{Protein expression and characterization}

The cDNAs corresponding to Ig I (amino acids 1-98), Ig II (amino acids 99-194), and Ig I-II (amino acids 1-194) from chicken N-CAM were generated by PCR from cDNA clones pEC208 and pEC254 [6] and cloned into the $N c o$ I and BamHI sites of the pET-3d vector (Novagen), and the resultant plasmids transformed into BL21 DE3 (PlysS) cells. The amino acid numbering is in accordance with that previously reported [2]. Protein expression was induced with $1 \mathrm{mM}$ IPTG at $18-25^{\circ} \mathrm{C}$ overnight. Ig I and Ig II were purified from the cell lysates by initial ammonium sulfate fractionation, followed by gel filtration and ion exchange chromatography. Ig I-II was purified from the cell membrane pellet by urea solubilization and gel filtration. The integrity of the expressed domains was established through sequencing of the cDNA, N-terminal sequencing of the first 10 amino acids of the purified proteins, and MALDI-TOF mass spectroscopy. Labeled proteins were produced from cells grown on minimal media, where uniformly labeled $\left[{ }^{15} \mathrm{~N}\right]$ ammonium sulfate and/or $\left[{ }^{13} \mathrm{C}\right]$ glucose 
were the sole sources of nitrogen and carbon, respectively. Protein concentrations were determined using the calculated extinction coefficients at $280 \mathrm{~nm}$ of $10095 \mathrm{M}^{-1} \mathrm{~cm}^{-1}, 8605 \mathrm{M}^{-1} \mathrm{~cm}^{-1}$, and 18700 $\mathrm{M}^{-1} \mathrm{~cm}^{-1}$ for Ig I, Ig II, and Ig I-II respectively [8].

Far UV circular dichroism (CD) spectra were recorded for each protein on an Aviv $60 \mathrm{DS}$ spectrometer at $20^{\circ} \mathrm{C}$ using a $0.1 \mathrm{~cm}$ pathlength cell. Spectra were averaged over three scans, and recorded from 260 to $190 \mathrm{~nm}$ using a time constant of $4 \mathrm{~s}$. The spectra were corrected for the contributions of buffers and were reported as mean residue ellipticities $\left(\mathrm{deg} \mathrm{cm}^{2} \mathrm{dmol}^{-1}\right)$

\subsection{NMR experiments}

NMR experiments were performed at $25^{\circ} \mathrm{C}$ (calibrated with neat methanol) on a Bruker DRX800 spectrometer equipped with a triple resonance probe and triple axis pulsed field gradients. ${ }^{1} \mathrm{H}-{ }^{15} \mathrm{~N}$ heteronuclear single quantum coherence (HSQC) spectra were acquired with 256 complex $t_{1}$ increments and 2048 complex data points in $t_{2}$. Quadrature detection in the indirect dimension was achieved with StatesTPPI [9]. Coherence selection was achieved using pulsed field gradients [10,11], and a water flip back pulse [12] used in addition to coherence selection to minimize the water resonance. Eight transients were recorded with sweep widths of $1161 \mathrm{~Hz}$ and $1914 \mathrm{~Hz}$ in the ${ }^{1} \mathrm{H}$ and ${ }^{15} \mathrm{~N}$ dimensions, respectively. Data sets were zero filled in each dimension and apodized with an $80^{\circ}$ shifted sine squared function using Felix (MSI), after initial conversion of the data in NMRPipe. The ${ }^{1} \mathrm{H}$ chemical shifts were referenced to TMS [13], and the ${ }^{15} \mathrm{~N}$ shifts referenced indirectly using the ratio of the gyromagnetic ratios [14].

\subsection{Ig I assignments}

Assignments for the backbone atoms were obtained from a ${ }^{13} \mathrm{C},{ }^{15} \mathrm{~N}$ labeled sample using standard triple resonance techniques, and will be presented elsewhere. The chemical shift index [15] was calculated from the $\mathrm{C} \alpha$ shifts using the random coil values of Wishart and Sykes [16].

\subsection{Binding studies}

The stock solutions used in the Ig I and Ig II binding experiments were A: $1.2 \mathrm{mM}{ }^{15} \mathrm{~N}$ Ig I, pH 7.0 in $90 \% \mathrm{H}_{2} \mathrm{O}, 10 \%{ }^{2} \mathrm{H}_{2} \mathrm{O}$, and $\mathrm{B}$ : $10.2 \mathrm{mM}$ Ig II, $1.2 \mathrm{mM}{ }^{15} \mathrm{~N}$ Ig I, pH 7.0 in $90 \% \mathrm{H}_{2} \mathrm{O}, 10 \%{ }^{2} \mathrm{H}_{2} \mathrm{O}$. HSQC spectra were recorded initially on solution $\mathrm{A}$, and after addition of aliquots of solution $\mathrm{B}$ up to a final concentration of $4.9 \mathrm{mM} \mathrm{Ig}$ II. The $\mathrm{pH}$ remained constant over the course of the titration. A final spectrum was collected after the addition of $12 \mu \mathrm{l}$ of $10 \mathrm{M} \mathrm{NaCl}$ to give a final $\mathrm{NaCl}$ concentration of $200 \mathrm{mM}$. An average $K_{\mathrm{d}}$ value was obtained by an iterative, non-linear fitting of the changes in the chemical shift $(\Delta)$ of 18 amide ${ }^{1} \mathrm{H}$ and ${ }^{15} \mathrm{~N}$ resonances of Ig I upon addition of Ig II, assuming a two-state second-order exchange process [17].

\subsection{Sedimentation experiments}

The sedimentation properties of Ig I-II in water were obtained from data collected on a temperature controlled Beckman XL-I analytical ultracentrifuge equipped with a An60Ti rotor and photoelectric scanner. A double sector cell, equipped with a $12 \mathrm{~mm}$ epon centerpiece and quartz windows, was loaded with 400-420 $\mu \mathrm{l}$ of sample using a blunt-end microsyringe for sedimentation velocity experiment. Data were collected at rotor speeds of 3000-50000 rpm in continuous mode at $20^{\circ} \mathrm{C}$ with a step size of $0.005 \mathrm{~cm}$ and an average of two scans per point. The data were then subjected to a direct boundary fitting using the Svedberg program (version 5.01) allowing for the calculation of the molecular weight of the sedimenting species using the $s$ (sedimentation coefficient) and $D$ (diffusion coefficient) values obtained from the fitting analysis [18].

Sedimentation equilibrium runs were performed on a $50 \mu \mathrm{M}$ Ig I-II sample $(110 \mu \mathrm{l})$ at $\mathrm{pH} 7$ and $22^{\circ} \mathrm{C}$, at speeds of $3000-14000 \mathrm{rpm}$ using a double sector cell with charcoal-filled epon centerpieces and sapphire windows. All scans were performed at $298 \mathrm{~nm}$, with a step size of $0.001 \mathrm{~cm}$ and 25 averaged scans. Samples were allowed to equilibrate for $24 \mathrm{~h}$, and duplicate scans $3 \mathrm{~h}$ apart were overlaid to determine that equilibrium had been reached. The data were analyzed by a non-linear least squares analysis using the Origin software provided by Beckman. The data were initially fitted to a single ideal species model using the following equation to determine the best fitting molecular weight:

$C_{\mathrm{r}}=\exp \left[\ln \left(C_{0}\right)+\left(M \omega^{2}(1-\bar{v} \rho) / 2 R T\right) \cdot\left(x^{2}-x_{0}^{2}\right)\right]+E$ where $C_{\mathrm{r}}$ is the concentration at radius $x, C_{0}$ is the concentration at a reference radius $x_{0}$ (usually the meniscus), $\bar{v}$ is the partial specific volume of the protein $(\mathrm{ml} / \mathrm{g}), \rho$ is the density of the solvent $(\mathrm{g} / \mathrm{ml})$, $\omega$ is the angular velocity of the rotor (radian/s), $E$ is the baseline error correction factor, $M$ is the molecular weight, $R$ is the universal gas constant $\left(8.314 \times 10^{7} \mathrm{erg} / \mathrm{mol}\right)$, and $T$ is temperature (Kelvin). The goodness of the fit was evaluated on the basis of the randomness and magnitude of the residuals, expressed as the difference between the theoretical curve and the experimental data, and evaluating the fit parameters. In the absence of a good fit to an ideal species, the data were fitted to a self-associating system of monomer to n-mer equilibria using:

$$
\begin{aligned}
C_{\mathrm{r}} & =\exp \left[\ln \left(C_{\text {monomer, } \mathrm{x} 0}\right)+\left(M \omega^{2}(1-\overline{\mathrm{v}} \rho) / 2 R T\right) \cdot\left(x^{2}-x 0^{2}\right)\right] \\
& +\exp \left[\operatorname{nln}\left(C_{\text {monomer, } \mathrm{x} 0}\right)+\ln \left(K_{\mathrm{a}, \mathrm{n}}\right) \cdot\left(n M(1-\overline{\mathrm{v}} \rho) \omega^{2} / 2 R T\right) \cdot\left(x^{2}-x_{0}^{2}\right)\right] \\
& +E
\end{aligned}
$$

where $K_{\mathrm{a}, \mathrm{n}}$ is the association constant for the formation of $n$-mer from monomer, and $M$ is the monomeric molecular weight.

\section{Results}

\subsection{Experiments with Ig I}

The N-terminal Ig-like domain of N-CAM, Ig I, expressed well in Escherichia coli and was readily purified. The CD spectrum of the purified protein showed a strong negative band centered at $217 \mathrm{~nm}$, consistent with the predominantly $\beta$-sheet character of an immunoglobulin domain (Fig. 1). Analysis of the well-dispersed ${ }^{1} \mathrm{H},{ }^{15} \mathrm{~N}$ HSQC spectrum confirmed that a single conformation was present in solution. Resonance assignments were determined for the majority of nuclei using standard triple resonance NMR techniques on a ${ }^{13} \mathrm{C},{ }^{15} \mathrm{~N}$ labeled domain (details will be presented elsewhere). Analysis of the spectra from $\mathrm{HNCACB}, \mathrm{CBCA}(\mathrm{CO}) \mathrm{NH}$, and $\mathrm{C}(\mathrm{CO}) \mathrm{NH}-\mathrm{TOCSY}$ experiments was sufficient to assign the protein domain. The chemical shift index (CSI) [15] calculated for the $\mathrm{C} \alpha$ carbons is presented in Fig. 2, and clearly shows

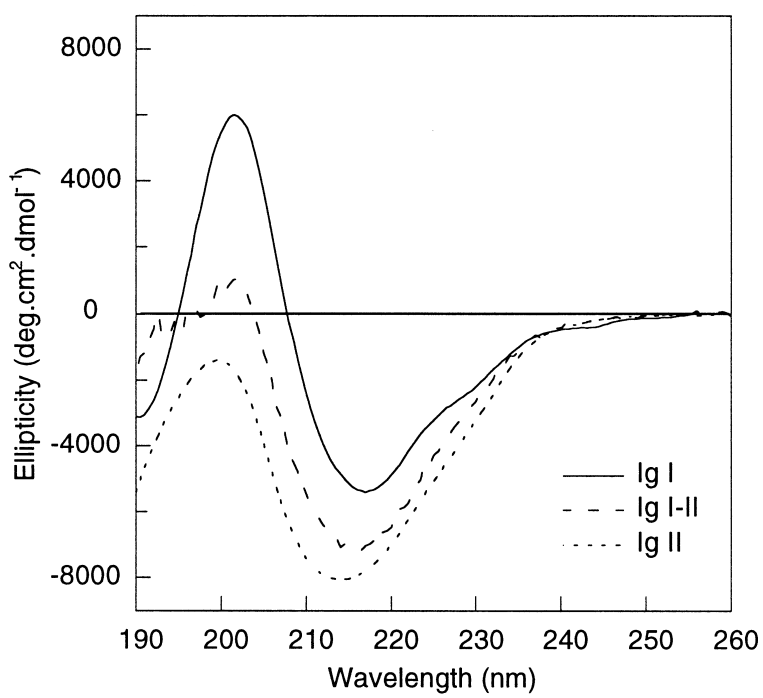

Fig. 1. Far UV circular dichroism spectra of Ig I (solid line, $5 \mu \mathrm{M}$ in water $\mathrm{pH}$ 6), Ig II (dotted line, $7 \mu \mathrm{M}$ water, $\mathrm{pH}$ 6) and Ig I-II (dashed line, $5 \mu \mathrm{M}$ in $60 \mu \mathrm{M}$ Tris, $\mathrm{pH}$ 7) recorded at $20^{\circ} \mathrm{C}$ on an Aviv 60 DS spectrometer. Spectra were recorded in a $0.1 \mathrm{~cm}$ pathlength cell, and were averaged over three scans with a time constant of $4 \mathrm{~s}$. Ellipticities are reported as mean residue ellipticity. 


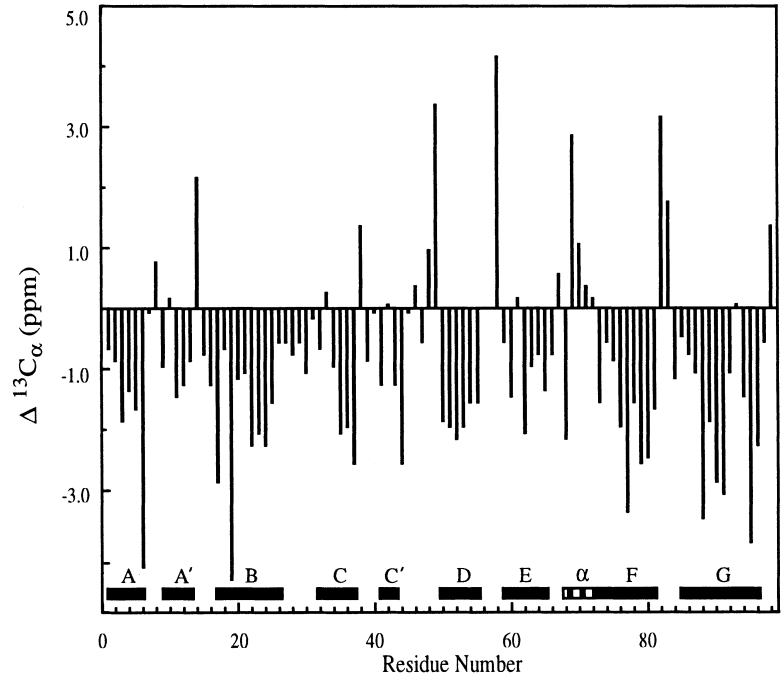

Fig. 2. $\mathrm{C}_{\alpha}$ chemical shift index (CSI) of the first Ig-like domain from chicken N-CAM, Ig I, plotted against residue number. The secondary structural elements delineated by the CSI are consistent with an immunoglobulin-like fold, and align with the $\beta$ sheets (solid bars) and the single turn of helix (striped bar) seen in murine Ig I [19]. The random coil chemical shifts of Wishart et al. [16] were used to calculate the CSIs.

the presence of multiple $\beta$ strands. The structure of Ig I from murine N-CAM determined by NMR [19] revealed an immunoglobulin fold from the intermediate or I-set, where the two $\beta$ sheets comprised strands ABED and $\mathrm{A}^{\prime} \mathrm{GFCC} \mathrm{C}^{\prime}$ and were linked by a disulfide bond. As expected from the sequence identity between chicken and mouse Ig I $(87 \%)$, the positions of the $\beta$ sheets and the single turn of helix correspond closely in the two proteins, confirming their identical folds (Fig. 2).

\subsection{Experiments with Ig II}

The protein expression level achieved for the second Ig-like domain, Ig II, was significantly lower than that found for Ig I, precluding the preparation of isotopically labeled material for direct NMR studies. The CD spectrum of Ig II displayed a negative peak centered around $215 \mathrm{~nm}$, consistent with a largely $\beta$ sheet protein and similar to that observed in Ig I (Fig. 1). The characteristic positive band around 195-200 nm associated with $\beta$ sheets was not observed. This may reflect subtle differences in the Ig folds between Ig I and Ig II. Alternatively, it may indicate less regular secondary structure in Ig II, as the amplitudes of the two observable CD bands for $\beta$ structure are dependent on the length of the sheets, their orientation, and the degree of twist [20].

\subsection{Ig I-Ig II titration}

The potential interaction of Ig I and Ig II was investigated by monitoring the ${ }^{1} \mathrm{H},{ }^{15} \mathrm{~N}$ HSQC spectrum of ${ }^{15} \mathrm{~N}$ Ig I on addition of unlabelled Ig II up to approximately 4 mol equivalents. All resonances were seen to broaden on addition of Ig II (Fig. 3), consistent with a change in the average transverse relaxation rate as a result of the formation of a higher molecular weight complex. In addition, differential broadening was observed for some resonances. In particular, the resonance of F19 broadened beyond detection at Ig II concentrations greater than $1 \mathrm{~mol}$ equivalent. Significant changes were also observed in the amide proton and nitrogen chemical shifts for a subset of Ig I resonances with increasing concentrations of Ig II (Fig. 3), but no new resonances were observed with increasing Ig II concentrations. The spectral changes observed during the titration, specifically the chemical shift changes of a subset of the resonances, the absence of any additional resonances, and the overall resonance broadening were consistent with the formation of a specific complex between Ig I and Ig II that was undergoing intermediate to fast exchange on the NMR timescale.

The most substantial changes in chemical shifts were seen for the resonances of S17, K18, F19, F20, L21 and I64, where the average change in the amide proton and nitrogen chemical shifts was $152 \mathrm{~Hz}$ as the Ig II concentration was increased up to $\sim 4$ mol equivalents. These residues are located at the $\mathrm{N}$ terminal end of the B strand (S17-L21) and the C-terminal end of the E strand (I64). As the B and E strands are apposed in the Ig fold, these residues describe a surface on one face of the molecule that represents a potential binding site. This site was further defined by smaller but still significant changes in the resonances of V6, C22, V52, L62 and Y65, and A67. These residues map to the $\mathrm{A}, \mathrm{B}, \mathrm{D}$ and $\mathrm{E}$ strands, and the EF loops respectively, and describe a region peripheral to the proposed binding site.

In addition, much smaller chemical shift changes (average $48 \mathrm{~Hz}$ ) were seen in resonances from G10 from the $\mathrm{A}^{\prime}$ strand, V14, G15, and E16 from the $\mathrm{A}^{\prime} \mathrm{B}$ loop, and N92 and K94 from the $\mathrm{G}$ strand. These residues define a second surface on the opposite face of the Ig fold, and are therefore unlikely to be directly involved in the binding of Ig II. These changes may reflect a small degree of non-specific binding. Alternatively, as this second surface is placed exactly behind the proposed Ig II binding site, and several of the residues are involved in the hydrophobic core of the domains, the observed changes may be a result of a slight conformational change in Ig I upon binding of Ig II, transmitted through the core of the domain. This suggestion is supported by the fact that the binding curves for these resonances show the same behavior as those at the proposed interaction site (see below).

Titration curves were generated for the 20 resonances where significant changes in the chemical shifts were observed, and clearly showed that the binding of Ig I and Ig II did not reach saturation (Fig. 4). The limited availability of Ig II prohibited collection of additional titration points at higher Ig II concentrations. An estimate of the dissociation constant, $K_{\mathrm{d}}$, was made by an iterative fitting of the changes in chemical shift in $\mathrm{Hz}$ on addition of Ig II, assuming a two-site second-order process in fast exchange [17]. A multi-parameter fit of 18 of the observed titrations to a single dissociation constant gave a value of $2.9 \pm 0.9 \mathrm{mM}$. Binding curves calculated with a $K_{\mathrm{d}}$ of $2.9 \mathrm{mM}$ for resonances from S17 and F20 are shown in Fig. 4, overlaid on the experimentally measured chemical shift changes.

The Ig I-Ig II titration was performed in the absence of any salts, and defined a largely hydrophobic interaction site on Ig I. To determine the role of electrostatic interactions in the observed interaction, $200 \mathrm{mM} \mathrm{NaCl}$ was added at the end of the titration. Minor chemical shift changes were seen in several resonances, including those from several residues in the proposed binding site (S17, F20, and I64) (data not shown). However, these changes were small compared to those attributed to the binding of Ig II, and there was no 


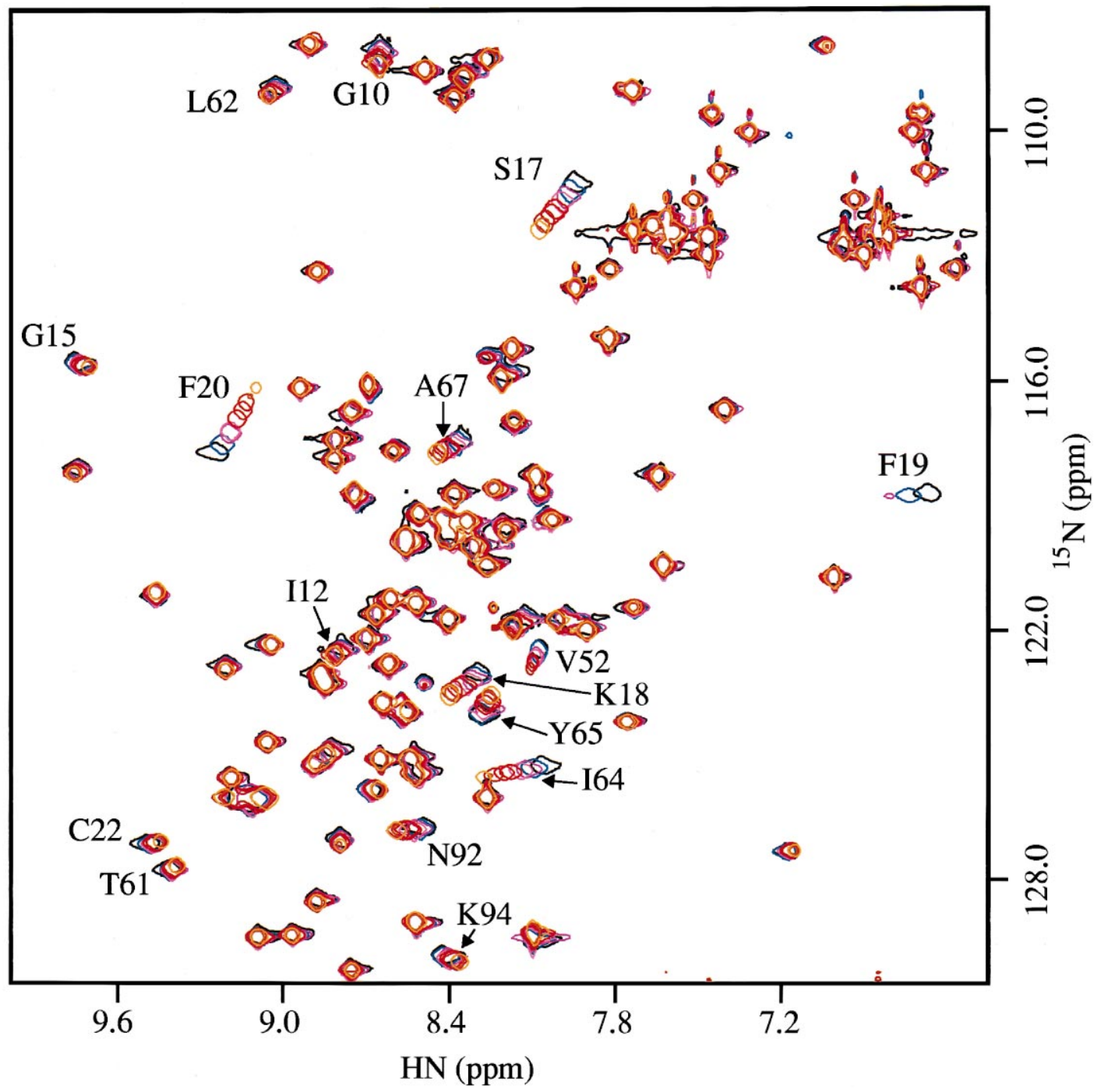

Fig. 3. ${ }^{1} \mathrm{H},{ }^{15} \mathrm{~N}$ HSQC spectra of $1.2 \mathrm{mM}{ }^{15} \mathrm{~N}$ Ig I at Ig II concentrations up to 4 molar equivalents. The spectrum of Ig I in the absence of Ig II is shown in black, while spectra at increasing Ig II concentrations are displayed in colors ranging from blue to yellow. Spectra were recorded in $90 \% \mathrm{H}_{2} \mathrm{O}, 10 \%{ }^{2} \mathrm{H}_{2} \mathrm{O}$ at pH $7.0,25^{\circ} \mathrm{C}$, on a Bruker DRX 800 spectrometer. The ${ }^{15} \mathrm{~N}$ spectral width of $1914 \mathrm{~Hz}$ resulted in the folding of the amide resonance of $\mathrm{L} 62$.

evidence of the reappearance of any unbound Ig I, suggesting that the Ig I-Ig II complex was not significantly disrupted.

\subsection{Experiments with $I g I-I I$}

The protein sequence corresponding to the first two Ig-like domains of N-CAM (Ig I-II) was expressed as a single polypeptide chain in E. coli. High levels of expression were achieved, but the Ig I-II protein was expressed in inclusion bodies. The protein was successfully recovered by solubilization in urea, and appeared to fold in the absence of denaturant. The CD spectrum recorded for Ig I-II showed the characteristic negative band centered about $216 \mathrm{~nm}$, but lacked the significant positive band around 195-200 nm normally associated with $\beta$ conformation. The spectrum corresponds closely to the sum of the spectra observed for the individual Ig-like domains, suggesting that any interdomain interactions that occur do not significantly perturb the conformations of the individual $\mathrm{Ig}$ folds.

Analytical ultracentrifugation was used to evaluate the oligomerization state of Ig I-II. In sedimentation velocity experi-
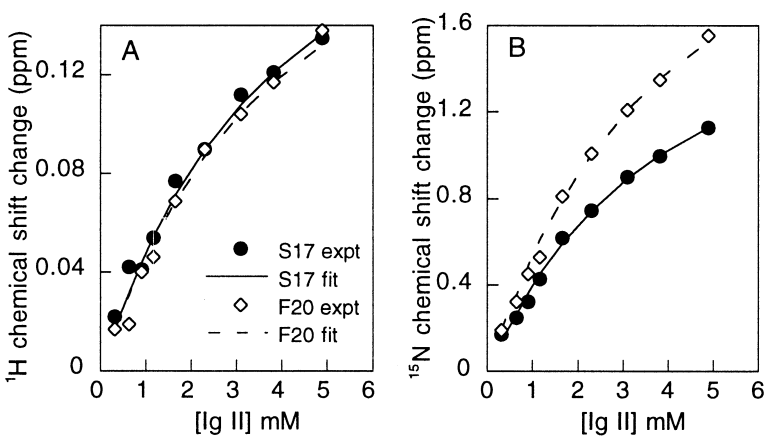

Fig. 4. Chemical shift titration curves for the ${ }^{1} \mathrm{H}$ (A) and ${ }^{15} \mathrm{~N}$ (B) resonances from S17 $(\bullet)$ and F20 $(\diamond)$ from ${ }^{15} \mathrm{~N}$ Ig I upon addition of Ig II. The titrations were fitted assuming a two site fast exchange regime to determine the $K_{\mathrm{d}}$ and the maximum change in chemical shift, $\Delta$. The experimental data are shown as symbols, and the fit of the relationship shown as a solid line for $\mathrm{S} 17$, and a dashed line for F20. 
ments Ig I-II (50 $\mu \mathrm{M}$ in $10 \mathrm{mM}$ Tris, $\mathrm{pH} 7.4)$ appeared to sediment as a 'single' boundary (Fig. 5A). Direct analysis of the data using the Svedberg program gave a reasonable fit to a single ideal species model yielding an $s$ value of $2.9 \mathrm{~S}$ and a diffusion coefficient $(D)$ of $7.1 \mathrm{~F}$, affording a molecular weight of $36.5 \mathrm{kDa}$. This value was less than the expected molecular weight of dimeric Ig I-II (45 kDa). Significantly better agreement between the experimental and theoretical data was achieved when a two species model was employed (Fig. 5A). The parameters obtained from the two species fit were $s 1=2.0$ $\mathrm{S}, D 1=8.6 \mathrm{~F}, c 1=0.143$ (AU) for the slow sedimenting species, and $s 2=2.9 \mathrm{~S}, D 2=6.6 \mathrm{~F}, c 2=1.08$ (AU) for the fast sedimenting species. This analysis yielded molecular weights of $22 \mathrm{kDa}$ and $41.7 \mathrm{kDa}$ for the slow and fast sedimenting species, respectively, and predicted a sample composition of roughly $12 \%$ monomer and $88 \%$ dimer, based on absorbance.
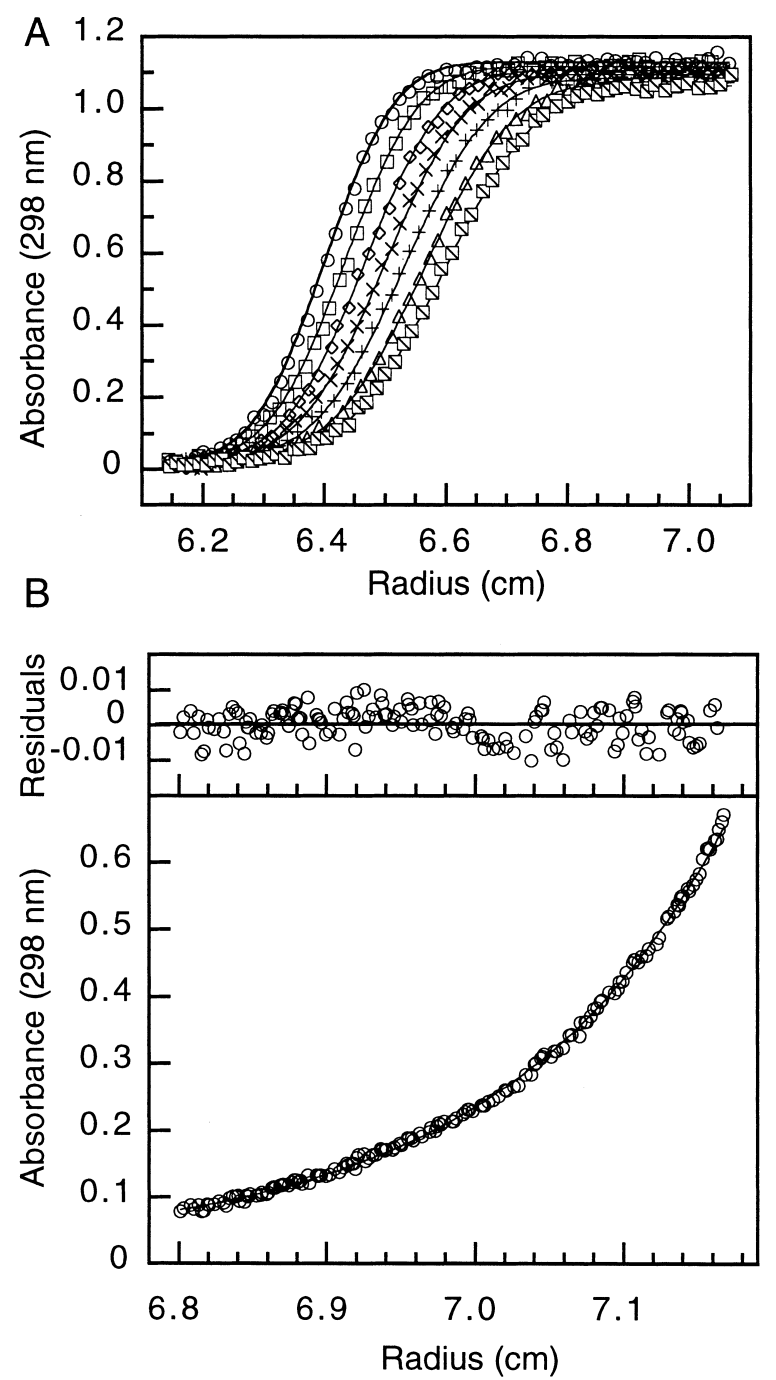

Fig. 5. Analytical ultracentrifugation data on $50 \mu \mathrm{M}$ Ig I-II in 10 $\mathrm{mM}$ Tris, $\mathrm{pH} 7.4$ and $0 \mathrm{mM}$ added $\mathrm{NaCl}$, collected on a Beckman XL I centrifuge. A: Overlay of successive sedimentation velocity profiles recorded at $\sim 10 \mathrm{~min}$ intervals, represented by different symbols, during an $50000 \mathrm{rpm}$ velocity experiment. The solid lines represent the direct fitting of the data to a two species model by the Svedberg program. B: Sedimentation equilibrium data recorded at $14000 \mathrm{rpm}(\mathrm{O})$ fitted to an associating model of monomer-dimer equilibrium (solid line). The residual differences between the experimental data and the fit for each point are shown above.
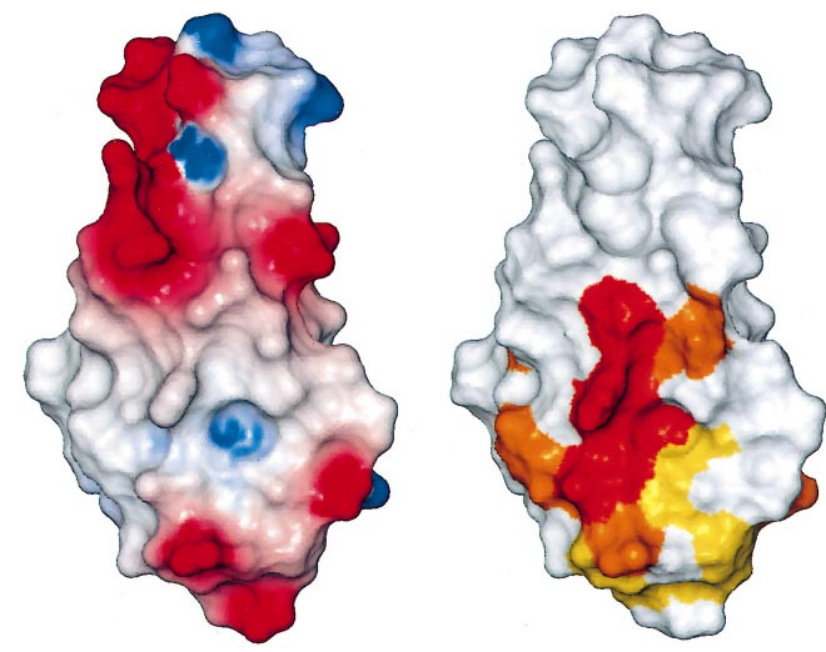

Fig. 6. Left image: Surface electrostatic potential for Ig I. The electrostatic potential was calculated using the simple charge approximation in the program MOLMOL, where charges are considered only on one or two atoms of the charged residue. A solvent radius of $2 \AA$, a salt concentration of $75 \mathrm{mM}$, and dielectric constants of 2 and 80 for the molecule and solvent, respectively, were used in the calculation. The gradient of electrostatic potential shown ranges from $-0.75 \mathrm{~K}_{\mathrm{B}} \mathrm{T} / \mathrm{e}$ (red) to $0.75 \mathrm{~K}_{\mathrm{B}} \mathrm{T} / \mathrm{e}$ (blue). Right image: Proposed Ig II binding surface on Ig I. The van der Waals surface of Ig I has been colored according to the sensitivity of the chemical shift of individual resonances to the interaction with Ig II. A vector describing the chemical shift deviations was calculated as $\left(\Delta_{\mathrm{H}}^{2}+\Delta_{\mathrm{N}}^{2}\right)^{1 / 2}$ where $\Delta$ is the chemical shift change in Hz. Residues for which the calculated vector was greater than 99 are colored red, 46-99 are colored orange, 30-46 are colored gold, and less than 30 are shown in white. This figure was prepared using the coordinates for murine Ig I [19] and the software MOLMOL [28].

Similar results were obtained in the presence of $100 \mathrm{mM}$ and $200 \mathrm{mM} \mathrm{NaCl}$ (data not shown).

In a sedimentation equilibrium study of Ig I-II in $10 \mathrm{mM}$ Tris, $\mathrm{pH}$ 7.4, a single ideal species model gave an average molecular weight of $38 \mathrm{kDa}$, but failed to fit the experimental data accurately, as judged by the residuals. Transforming the equilibrium concentration gradient (absorbance vs. radius) into a $M_{\mathrm{w}, \mathrm{app}}$ versus concentration plot revealed a molecular weight distribution between that of a monomer and a dimer (32-38 kDa), suggesting a monomer-dimer equilibrium. Fitting the data to an associating model of monomer-dimer equilibrium gave an excellent fit, as judged by the residuals (Fig. 5B). Sedimentation equilibrium studies were also carried out in the presence of $100 \mathrm{mM}$ and $200 \mathrm{mM} \mathrm{NaCl}$ to evaluate the effect of salt on the Ig I-II association. Attempts to fit the data collected in the presence of salt to a single species also failed, but excellent agreement was achieved with a self-associating model of monomer-dimer equilibrium. Estimates of the dissociation constants $\left(K_{\mathrm{d}}\right)$ were determined as $91 \mu \mathrm{M}$, $94 \mu \mathrm{M}$, and $102 \mu \mathrm{M}$ for $0 \mathrm{mM}, 100 \mathrm{mM}$, and $200 \mathrm{mM}$ $\mathrm{NaCl}$ respectively. The experimental data from both the sedimentation velocity and the sedimentation equilibrium experiments clearly demonstrated that Ig I-II exists in equilibrium between monomer and dimer, and that the association state is independent of salt concentration.

\section{Discussion}

The characterization of the molecular mechanisms of cell 
adhesion is challenging, because not only are the interactions weak between membrane proteins, but also because such interactions can be cis (between molecules on the same cell) or trans (apposing cells), as well as homo- or heterophilic. Models for the interactions of some adhesion proteins, for example the myelin $\mathrm{P}_{0}$ [21] and the $\mathrm{Ca}^{2+}$ dependent $\mathrm{E}$ cadherin [22], have been proposed based on the similarly weak interactions observed in crystal structures. No crystal structures are available for any of the domains of N-CAM, and none of the models proposed for N-CAM mediated cell adhesion [5$7,23]$ is compatible with all of the current experimental findings.

In this work, we have used NMR to study the binding of the first two Ig-like domains from N-CAM expressed as independent, soluble proteins, and found a weak, but specific interaction. The observed chemical shift changes of resonances defined a potential binding site on Ig I, centered on the B and $\mathrm{E} \beta$ strands. The amino acids involved in the proposed Ig I-Ig II binding site are conserved across species, thereby allowing the proposed site to be displayed on the determined murine Ig I structure (Fig. 6). The sensitivity of a residue's chemical shift to Ig II binding is shown using a gradation of colors, where the most affected residues are shown in red. The titrating residues map to a largely uncharged surface on $\mathrm{Ig} \mathrm{I}$, implying that the binding is dominated by hydrophobic interactions. The side chain of K18, the single charged amino acid in the defined surface, is involved in the hydrophobic core, with the amino group forming a salt bridge with the carboxylate of D71 that lies in the single turn of helix between strands E and F [19]. In addition, the side chain of F20 participates in the formation of the hydrophobic core, and the side chain of I64 is oriented towards the center of the molecule. The surface accessible side chains in the proposed binding site include those of S17 and T63, and those of F19 and Y65.

An interaction between the first two Ig-like domains of murine N-CAM has been previously shown by surface plasmon resonance [7], and a model for this interaction derived based on the determined structure for Ig I and a modeled structure for Ig II [19]. In these studies, it was proposed that an antiparallel interaction of the Ig domains was driven by surfaces of complementary charge on the two domains, where acidic side chains located at the N-terminal end of Ig I interacted with negatively charged side chains predicted to be at the C-terminal end of Ig II [19]. This model differs from our findings, which identify a hydrophobic interaction site. Support for a hydrophobic rather than an electrostatically driven interaction was found in the insensitivity of the chemical shifts of the proposed interacting acidic residues in Ig I on addition of Ig II, and the minimal effects observed on addition of salt to the Ig I-Ig II complex.

To further characterize the interaction of Ig I with Ig II, we expressed the two domains as a single polypeptide chain with the native linker sequence. The $\mathrm{CD}$ data indicate that there are no significant changes in the conformation of the individual domains when they are expressed together as a concatemer. Analytical ultracentrifugation studies show that the Ig I-II protein exists predominantly as a dimer at micromolar concentrations, and that the association is essentially independent of the concentration of $\mathrm{NaCl}$, up to $200 \mathrm{mM}$. The significantly stronger association observed for the covalently linked domains ( $\sim 100 \mu \mathrm{M}$ compared to $\sim 3 \mathrm{mM}$ ) is therefore consistent with the reciprocal binding of the Ig I and Ig II domains arranged in an antiparallel orientation.

The dissociation constant for the Ig I-Ig II binding measured using surface plasmon resonance techniques [7] was significantly stronger than that determined here, and, in fact, is similar to the affinity measured for the Ig I-II concatemer; $55 \mu \mathrm{M}, \sim 3 \mathrm{mM}, \sim 100 \mu \mathrm{M}$ respectively. This discrepancy may be due to the use of Ig II as a fusion with maltose binding protein in the surface plasmon resonance experiments [7].

The well characterized dimerization of immunoglobulin domains in antibodies involves homologous surfaces of like domains, that is, the constant domains interact via close packing of the ABED faces, while variable domains interact through the surfaces formed by the $\mathrm{GFCC}^{\prime} \mathrm{C}^{\prime \prime}$ strands. For the I-set intercellular adhesion molecule 1, ICAM-1, thought to exist as a dimer on the cell surface, a dimerization model proposes interactions of residues on the BED face in a cis orientation [26]. In contrast, both faces of the $\beta$ sandwich are utilized in the dimerization of the I-set domains of hemolin, an insect immunoglobulin. The four I-set domains of hemolin fold into a novel horseshoe arrangement, where the antiparallel domain 1-domain 4 and domain 2-domain 3 interactions are mediated via the GFC and the ABED sheets respectively [27]. It appears that the dimerization of the I-set domains is more permissive, proceeding through either face of the $\beta$ sandwich, but still involving homologous surfaces. Thus, it is predicted that the interaction of N-CAM Ig I with Ig II will be centered on the ABED face of Ig II.

The findings presented here, and those reported from others [7], support a specific interaction of the first two Ig-like domains of N-CAM. Despite differences in the nature of the proposed interaction surfaces, both studies propose an antiparallel orientation of Ig I and Ig II. Binding studies of individually expressed Ig domains adsorbed onto a solid surface [6], and cell based adhesion studies of chimeric proteins [5] have implicated all five Ig-like domains of N-CAM in homophilic binding. Furthermore, the level of glycosylation on the fifth Ig-like domain of N-CAM is known to affect cell adhesion [3]. Thus, a simple homophilic binding model involving only interactions between the first two Ig-like domains does not fully account for the experimental evidence. N-CAM mediated cell adhesion is known to be concentration dependent, implicating cis as well as trans interactions as important. Although the binding affinities estimated from the Ig I-Ig II titration and the ultracentrifugation studies on Ig I-II are of the order of magnitude expected for cell adhesion complexes, it is not possible to say whether the observed Ig I-Ig II interaction is central to N-CAM homophilic binding. Despite these caveats, the ability of the Ig I-Ig II complex to survive the addition of physiological concentrations of salt suggested not only that the interaction was mediated largely by hydrophobic contacts, but that the complex was sufficiently robust to exist under conditions expected in the extracellular environment.

Acknowledgements: We thank Adrian Badillo, Adriana Aguilera, and Shaun Sheets for excellent technical assistance, and John Chung and Brendan Duggan for help with NMR experiments. The analytical ultracentrifuge measurements were made with the cooperation of Dr. Jeffrey Kelly. This work was supported by NIH grants HD 16550 (B.A.C.), HD 09635 (G.M.E.) and CA 27489 (H.J.D. and P.E.W.). 


\section{References}

[1] Brummendorf, T. and Rathjen, F.G. (1996) Curr. Opin. Neurobiol. 6, 584-593.

[2] Cunningham, B.A., Hemperly, J.J., Murray, B.A., Prediger, E.A., Brackenbury, R. and Edelman, G.M. (1987) Science 236, 799-806.

[3] Cunningham, B.A., Hoffman, S., Rutishauser, U., Hemperly, J.J. and Edelman, G.M. (1983) Proc. Natl. Acad. Sci. USA 80, 31163120 .

[4] Watanabe, M., Frelinger, A.L. and Rutishauser, U. (1986) J. Cell Biol. 103, 1721-1727.

[5] Zhou, H., Fuks, A., Alcaraz, G., Bolling, T.J. and Stanners, C.P. (1993) J. Cell Biol. 122, 951-960.

[6] Ranheim, T.S., Edelman, G.M. and Cunningham, B.A. (1996) Proc. Natl. Acad. Sci. USA 93, 4071-4075.

[7] Kiselyov, V.V., Berezin, V., Maar, T.E., Soroka, V., Edvardsen, K., Schousboe, A. and Bock, E. (1997) J. Biol. Chem. 272, $10125-10134$.

[8] Pace, C.N., Vajdos, F., Fee, L., Grimsley, G. and Gray, T. (1995) Protein Sci. 4, 2411-2423.

[9] Marion, D., Ikura, M., Tschudin, R. and Bax, A. (1989) J. Magn. Reson. 85, 393-399.

[10] Kay, L.E., Keifer, P. and Saarinen, T. (1992) J. Am. Chem. Soc. 114, 10663-10665.

[11] Muhandiram, D.R. and Kay, L.E. (1994) J. Magn. Reson. Ser. B 103, 203-216.

[12] Grzesiek, S. and Bax, A. (1993) J. Am. Chem. Soc. 115, 12593 12594.

[13] Orbons, L.P., van der Marel, G.A., van Boom, J.H. and Altona, C. (1987) Eur. J. Biochem. 170, 225-239.

[14] Wishart, D.S., Bigam, C.G., Yao, J., Abildgaard, F., Dyson,
H.J., Oldfield, E., Markley, J.L. and Sykes, B.D. (1995) J. Biomol. NMR 6, 135-140.

[15] Wishart, D.S. and Sykes, B.D. (1994) Methods Enzymol. 239, 363-392.

[16] Wishart, D.S., Bigam, C.G., Holm, A., Hodges, R.S. and Sykes, B.D. (1995) J. Biomol. NMR 5, 67-81.

[17] Lian, L.-Y. and Roberts, G.C.K. (1993) in: NMR of Macromolecules. A Practical Approach (Roberts, G.C.K., Ed.), pp. 153 182, Oxford University Press, Oxford.

[18] Philo, J.S. (1997) Biophys. J. 72, 435-444.

[19] Thomsen, N.K., Soroka, V., Jensen, P.H., Berezin, V., Kiselyov, V.V., Bock, E. and Poulsen, F.M. (1996) Nature Struct. Biol. 3, 581-585.

[20] Manning, M.C., Illangasekare, M. and Woody, R.W. (1988) Biophys. Chem. 31, 77-86.

[21] Shapiro, L., Doyle, J.P., Hensley, P., Colman, D.R. and Hendrickson, W.A. (1996) Neuron 17, 435-449.

[22] Nagar, B., Overduin, M., Ikura, M. and Rini, J.M. (1996) Nature 380, 360-364.

[23] Rao, Y., Zhao, X. and Siu, C.H. (1994) J. Biol. Chem. 269 27543-27548.

[24] McAlister, M.S.B., Mott, H.R., van der Merwe, P.A., Campbell, I.D., Davis, S.J. and Driscoll, P.C. (1996) Biochemistry 35, 5982 5991.

[25] Grzesiek, S., Stahl, S.J., Wingfield, P.T. and Bax, A. (1996) Biochemistry 35, 10256-10261.

[26] Casasnovas, J.M., Springer, T.A., Liu, J., Harrison, S.C. and Wang, J. (1997) Nature 387, 312-315.

[27] Su, X.D., Gastinel, L.N., Vaughn, D.E., Faye, I., Poon, P. and Bjorkman, P.J. (1998) Science 281, 991-995.

[28] Koradi, R., Billeter, M. and Wüthrich, K. (1996) J. Mol. Graph. $14,51-55$. 